\title{
Variabilidade físico-química da farinha de mandioca do Território da Cidadania do Vale do Juruá, Acre ${ }^{1}$
}

\author{
Virgínia de Souza Álvares ${ }^{2}$, Daniela Popim Miqueloni ${ }^{3 *}$, Jacson Rondinelli da Silva Negreiros ${ }^{4}$
}

$10.1590 / 0034-737 X 201663020001$

\begin{abstract}
A farinha é um derivado da mandioca de grande importância alimentar, porém com pequena padronização, por causa do processo artesanal de fabricação. O objetivo deste estudo foi analisar a variabilidade da farinha de mandioca artesanal, produzida no Território da Cidadania do Vale do Juruá, Acre, e agrupar os municípios produtores de acordo com suas características físico-químicas, por meio de análises multivariadas, determinando sua influência na qualidade da farinha de mandioca. Foram analisadas 138 amostras de farinhas, coletadas nos municípios de Cruzeiro do Sul, Mâncio Lima, Rodrigues Alves, Porto Walter e Marechal Thaumaturgo, com determinação da umidade, cinzas, proteína total, extrato etéreo, fibra total, carboidratos totais, valor energético, acidez titulável, $\mathrm{pH}$ e atividade de água. Os dados foram analisados pela estatística descritiva com comparação de médias pelo teste de Tukey e estatística multivariada, de forma complementar entre si; com análises de agrupamento hierárquica, pela distância euclidiana e método de Ward, e, não hierárquica, k-means, análise de componentes principais, pela matriz de correlação, e análise discriminante, pelo método da exclusão progressiva passo a passo. Os resultados mostraram que as farinhas encontram-se dentro das normas de qualidade exigidas em legislação. As diferentes análises multivariadas foram coerentes, indicando que há um padrão de distribuição das características físico-químicas das farinhas, o que sugere padrões no processo de fabricação, distribuídos conforme a localização dos municípios analisados. As características de maior influência na discriminação das farinhas são acidez, pH, atividade de água e umidade, indicando que o modo de fabricação tem grande influência na qualidade da farinha produzida.
\end{abstract}

Palavras-chave: produto da mandioca, artesanal, análise multivariada.

\section{RESUMO}

\section{Physico-chemical variability of cassava flour in the Território da Cidadania do Vale do Juruá, Acre}

The flour is a processed cassava food of great dietary importance, but with little standardization due to the craft manufacturing process. The aim of this study was to analyze the variability of artisanal cassava flour produced in Território da Cidadania do Vale do Juruá, Acre, and group the producing municipalities according to physico-chemical characteristics using multivariate analysis todetermine their influence on the flour quality. A total of 138 flour samples were collected in the cities of Cruzeiro do Sul, Mâncio Lima, Rodrigues Alves, Porto Walter and Marechal Thaumaturgo. Moisture, ash, total protein, ether extract, total fiber, total carbohydrate, energy value, titratable acidity, $\mathrm{pH}$ and water activity were determined in the samples. Data were analyzed using descriptive statistics and means compared with the Tukey test. and multivariate statistics in a complementary way, hierarchical cluster analysis with Euclidean distance and Ward method, and non-hierarchical, k-means, principal components analysis with correlation matrix, and backward

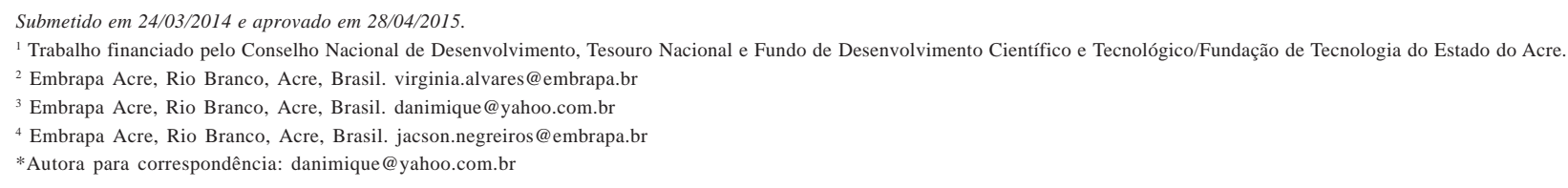


stepwise discriminant analysis. The results showed that the flours met the quality standards required by the legislation. The different multivariate analyzes were consistent, indicating that the physico-chemical properties of the flours have a pattern of distribution, which suggests standards in the manufacturing process distributed according to the location of the municipalities analyzed. The most influencing characteristics for flour discrimination are acidity, $\mathrm{pH}$, water activity and moisture, indicating that the mode of production has great influence on the quality of the flour produced.

Key words: cassava, craft food, multivariate analysis.

\section{INTRODUÇÃO}

A mandioca, com provável origem no sul da Bacia Amazônica, é um alimento com alto teor energético, gerando diversos subprodutos, como farinha das raízes e folhas, amidos e fécula (Chisté et al., 2006; Chisté \& Cohen, 2011; Fiorda et al., 2013). A farinha, com consumo generalizado, no Brasil, é um produto tipicamente brasileiro, que não encontra equivalente, a não ser na África, chegando a constituir a base alimentar em muitos estados, principalmente do norte e nordeste do país (Cereda \& Vilpoux, 2010; Dósea et al., 2010; Oluwamukomi et al., 2011).

O processo básico de fabricação da farinha compreende as etapas de lavagem, descascamento, trituração, prensagem e torração, variando de acordo com os tipos de farinha e as localidades (Chisté \& Cohen, 2006; Oluwamukomi et al., 2011). No entanto, por causa da baixa padronização do processo artesanal de fabricação, que consiste basicamente em processos próprios das casas de farinha locais, sua valorização e produção industrial são baixas (Souza et al., 2008a).

Por essa razão, a variabilidade dos tipos artesanais de farinha é alta e não permite sua comercialização de forma padronizada no país, concentrando-se os polos produtores nos mercados consumidores locais (Ferreira Neto et al., 2004). Além disso, a classificação da farinha muitas vezes é particular de cada fabricante, abrangendo desde a granulação até a cor do produto final (Velthem \& Katz, 2012), conferindo à qualidade da farinha de mandioca, em muitos locais, um caráter subjetivo (Cereda \& Vilpoux, 2010). Fatores como cultivar, clima, solo, colheita, entre outros, também são responsáveis por esta variabilidade (Fiorda et al., 2013). Contudo, o método de processamento é apontado como principal fator responsável pela qualidade da farinha, no qual as etapas de processamento, como torrefação e prensagem, são de grande influência no produto final (Dias \& Leonel, 2006; Cereda \& Vilpoux, 2010).

As farinhas do Estado do Acre já foram objeto de estudo (Souza et al., 2008a; 2008b; Velthem \& Katz, 2012; Álvares et al., 2013), indicando que, na maioria dos casos, encontram-se dentro dos padrões de qualidade exigidos pela legislação. Contudo, estudadas separadamente, por regiões menores, foram encontrados apenas padrões nos processos de produção da farinha. Considerando-se estes fatores, a determinação da qualidade e a classificação das farinhas de mandioca em uma ampla visão geográfica tornam-se processos complexos, que necessitam de ferramentas objetivas e confiáveis no estabelecimento das análises. Por isso, a análise multivariada apresenta grande potencial de análise e estruturação dos dados, com resultados complementares e capazes de captar padrões muitas vezes invisíveis a outras análises (Mingoti, 2007).

O objetivo deste estudo foi analisar a variabilidade da farinha de mandioca artesanal produzida no Território da Cidadania do Vale do Juruá, Acre, e agrupar os municípios produtores, de acordo com suas características físico-químicas, determinando sua influência na qualidade da farinha de mandioca por meio de análises multivariadas.

\section{MATERIAL E MÉTODOS}

Foram coletadas 138 amostras de farinha de mandioca, em casas de farinha com produção artesanal, do Território da Cidadania do Vale do Juruá, Acre, que abrange os municípios de Cruzeiro do Sul, com 48 amostras, Mâncio Lima, 16 amostras, Rodrigues Alves 29 amostras, Porto Walter 21 amostras, e Marechal Thaumaturgo, 24 amostras (Figura 1), de Janeiro a Dezembro de 2009, com a finalidade de determinar as características físico-químicas dessas farinhas. $\mathrm{O}$ tempo entre a coleta de amostras e as análises laboratoriais variou, de acordo com a logística de coleta e a distância do local de análise, sendo de 24 a 48 h para os municípios mais próximos (Cruzeiro do Sul, Mâncio Lima e Rodrigues Alves), até uma semana, para os mais distantes (Marechal Thaumaturgo e Porto Walter). As amostras foram acondicionadas em sacos plásticos e encaminhadas para o Laboratório de Tecnologia de Alimentos da Empresa Brasileira de Pesquisa Agropecuária (Embrapa), em Rio Branco, AC.

\section{Caracterização físico-química}

A caracterização físico-química das farinhas compreendeu a determinação, em percentagem (\%), de umidade $(\mathrm{U})$, cinzas $(\mathrm{CZ})$, extrato etéreo (EE), proteína total (P), fibra bruta total (F) e carboidratos (C), bem como acidez titulável, 
valor energético (VE), pH e atividade de água (Aw). A umidade foi determinada em estufa, a $105^{\circ} \mathrm{C}$, por oito horas. $\mathrm{O}$ teor de cinzas (CZ) foi obtido com a carbonização das amostras, até o fim da liberação de fumaça, e calcinação em mufla, a $540{ }^{\circ} \mathrm{C}$, até peso constante. Já o extrato etéreo (EE) foi obtido por extração em Soxhlet, durante dez horas, seguido por evaporação do solvente. O teor de proteína total (P) foi verificado pela técnica de micro-Kjeldahl, com hidrólise e posterior destilação da amostra, utilizando-se o fator $6,25 \times \%$ N. O teor de fibra bruta $(\mathrm{F})$ foi determinado por meio de digestão do material, em solução de $\mathrm{H}_{2} \mathrm{SO}_{4}$ a $1,25 \%$ p/v, por 30 minutos, seguida de $\mathrm{NaOH} \mathrm{1,25 \%} \mathrm{m/v,}$ por mais 30 minutos (AOAC, 1995). Já a acidez titulável (Acidez), em meq $\mathrm{NaOH} \cdot 100^{-1} \mathrm{~g}$, foi verificada por titulometria, utilizando-se $\mathrm{NaOH} 0,1 \mathrm{~N}$. Os carboidratos totais $(\mathrm{C})$, em \%, foram estimados por diferença, subtraindose de 100 o somatório de proteínas, extrato etéreo, cinzas, umidade e fibra bruta, segundo as Normas Analíticas do Instituto Adolfo Lutz (2008). O valor energético (VE) foi calculado a partir dos coeficientes calóricos correspondentes, para proteínas, extrato etéreo e carboidratos, respectivamente 4,9 e $4 \mathrm{kcal} \mathrm{g}^{-1}$, segundo Anvisa (Brasil, 2001) e o resultado foi expresso em kcal $100 \mathrm{~g}^{-1}$. O pH foi obtido, utilizando-se nove gramas da amostra, os quais foram misturadas em $60 \mathrm{~mL}$ de água destilada, homogeneizados e deixados em repouso, por 30 minutos, sendo a leitura feita em medidor digital. A atividade de água (Aw) foi determinada em medidor de atividade de água portátil, modelo Pawkit, marca Decagon.

\section{Análises estatísticas}

Os dados obtidos das amostras de farinhas, selecionadas inteiramente ao acaso e com três repetições por amostra em cada casa de farinha, foram analisados, segundo procedimentos da estatística descritiva, referentes às medidas de tendência central e variabilidade pelo teste de normalidade de Kolmogov-Smirnov a 5\%.
Com o intuito de detectar uma possível estrutura de grupos, quanto às características físico-químicas das farinhas, os dados foram submetidos às análises de agrupamento, por métodos hierárquicos e não hierárquicos. A análise por método hierárquico foi processada, considerando-se a distância euclidiana simples, e o método de Ward para definição de um número definido de grupos para o processamento complementar da análise por método não hierárquico, utilizando-se o algoritmo k-means. As médias dos dados obtidos para cada grupo foram analisadas pelo teste de Tukey, a 5\% de probabilidade.

Posteriormente, a análise de componentes principais (ACP) foi processada com o objetivo de discriminar, em poucas dimensões, as farinhas, segundo as propriedades físico-químicas. Utilizando-se os autovalores acima de um, conforme critério de Kaiser, obtidos da matriz de covariância dos dados originais, foram construídas novas variáveis latentes (autovetores), denominados de componentes principais, que são combinações lineares das variáveis originais medidas (Mingoti, 2007). Para estas análises, os dados foram padronizados, de forma a obter-se média nula e variância unitária. Os autovalores representam o comprimento dos eixos das componentes principais das variáveis com unidade de variância. Para cada autovalor, existe um vetor de módulo unitário, chamado autovetor, que representa a direção dos eixos das componentes principais que podem ser empregados como índices para classificar amostras de farinha da área, em um intervalo de valores determinados pelos componentes principais (Mingoti, 2007). Estas técnicas multivariadas são consideradas de interdependência, sem a normalidade como requisito para as análises de agrupamento e componentes principais.

Em seguida, a análise discriminante foi utilizada como técnica de dependência, considerando-se como variáveis categóricas os municípios produtores das farinhas. Essa análise estima a combinação linear das características dos

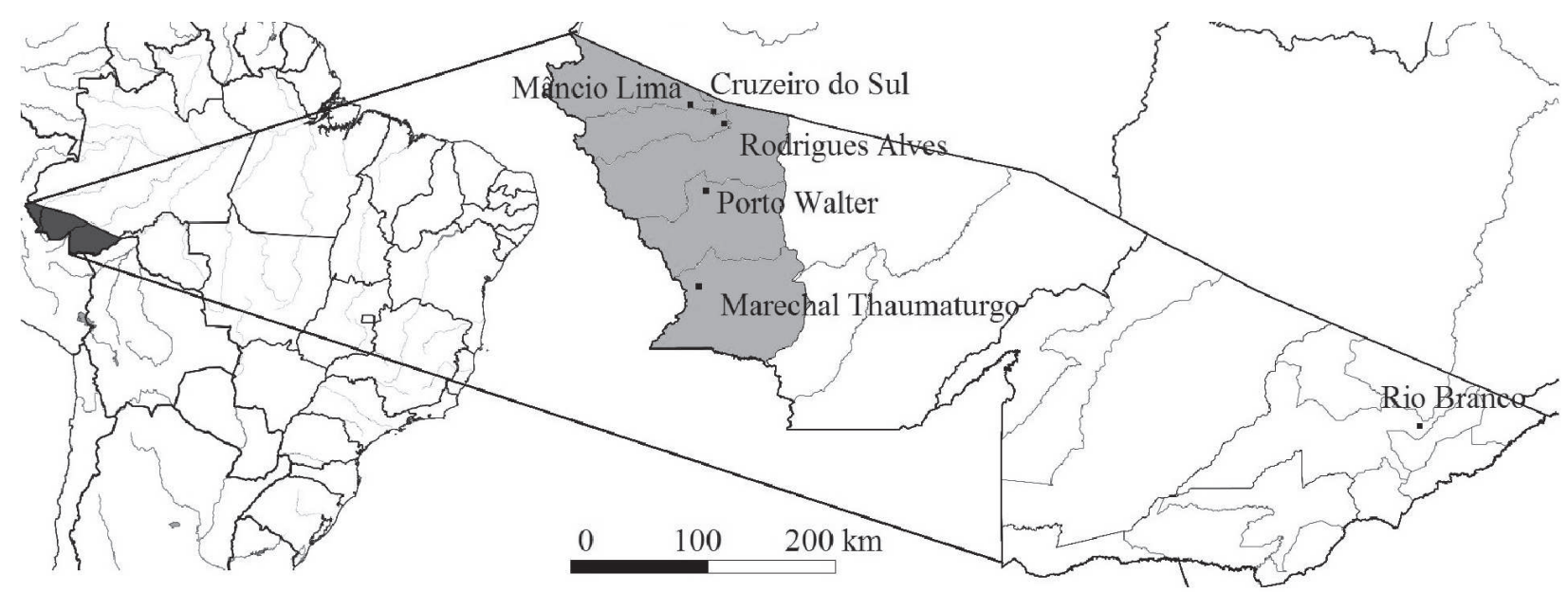

Figura 1: Localização dos municípios de coleta no Estado do Acre, em relação à capital, Rio Branco. 
elementos que melhor discriminam os grupos (Mingoti, 2007). Foi utilizado o método de exclusão progressiva passo a passo (backward stepwise), gerando um modelo discriminante, formado por duas variáveis ortogonais de máximo poder discriminante. Destes modelos, foram geradas a matriz de classificação e as funções discriminantes e testadas as correlações entre as variáveis físico-químicas de maior relevância, obtidas na análise de componentes principais. Estas variáveis foram transformadas de modo a se aproximarem da distribuição normal.

O uso das diversas análises multivariadas deu-se com o intuito de complementar os possíveis resultados, gerando interpretações para a próxima etapa de análises, como os agrupamentos, ou corroborando o resultado da análise anterior, como as análises discriminante e de componentes principais. Todas as análises estatísticas foram processadas pelo programa Statistica 7.0 (Statsoft, 1995).

\section{RESULTADOS E DISCUSSÕES}

Segundo a Legislação Brasileira para farinha de mandioca (Brasil, 2011), os valores médios observados para as variáveis físico-químicas das amostras de farinha encontram-se de acordo com os padrões estabelecidos para os teores de umidade, de cinzas e de fibras, com máxima de $13 \%$ de umidade, $1,4 \%$ para cinzas e $2,3 \%$ para fibras (Tabela 1), o que também foi observado por Souza et al. (2008a; 2008b), mas não por Álvares et al. (2013) para farinhas da mesma região. Ainda de acordo com esta legislação, de modo geral, as farinhas analisadas apresentam acidez baixa $\left(\leq 3,0\right.$ meq NaOH.100 g $\left.\mathrm{g}^{-1}\right)$, já esperada para uma farinha do Grupo Seca, o que não foi observado por Dias \& Leonel (2006) e Chisté et al. (2006), que obtiveram valores superiores aos permitidos pela legislação, ao avaliarem diferentes farinhas de mandioca.
Com base na variabilidade das características físico-químicas, a estruturação de classes das farinhas, por meio da análise de agrupamento hierárquica, sugeriu a formação de 4 grupos (Figura 2). O grupo A, formado principalmente por amostras do município de Marechal Thaumaturgo, destaca-se com os maiores valores de umidade, acidez, carboidratos, extrato etéreo, $\mathrm{pH}$ e atividade de água. Segundo Chisté \& Cohen (2011), a umidade está relacionada com fatores do processo de fabricação da farinha, principalmente à torração do produto, como tempo, temperatura do forno e eficiência do processo de prensagem; bem como a acidez, que também pode ser alterada por interrupção do processo de fabricação e pela fermentação (Chisté \& Cohen, 2007). O grupo C é formado por amostras de Porto Walter e Cruzeiro do Sul, com valores medianos das características físico-químicas, exceto pelos maiores teores de cinzas e fibras, assim como os grupos B e D. No entanto, estes grupos foram menos homogêneos, com amostras obtidas em Mâncio Lima, Cruzeiro do Sul e Rodrigues Alves. Os diferentes teores de cinzas e fibras podem estar relacionados com as características genéticas dos cultivares de mandioca utilizados, além das características edafoclimáticas, como observado por Fiorda et al. (2013), na produção de fécula de mandioca. Além disso, é possível observar a tendência de formação de grupos de acordo com a distribuição geográfica, segundo os métodos de produção familiar (Velthem \& Katz, 2012). Já Souza et al. (2008a) atribuem essa variação das características das farinhas ao processo de produção, porém analisando apenas as farinhas oriundas da região de Cruzeiro do Sul.

A partir da formação desses quatro grupos, a análise não hierárquica k-means foi conduzida de forma complementar, resultando no gráfico de perfil dos centroides, isto

Tabela 1: Estatística descritiva global e valores médios por grupo segundo método hierárquico dos dados físico-químicos das amostras de farinha de mandioca do Território da Cidadania do Vale do Juruá, Acre

\begin{tabular}{|c|c|c|c|c|c|c|c|c|c|c|}
\hline Variáveis & $\mathbf{U}$ & $\mathbf{C Z}$ & $\mathbf{P}$ & $\mathbf{E E}$ & $\mathbf{F}$ & $\mathbf{C}$ & $\mathbf{V E}$ & Acidez & pH & Aw \\
\hline Média & 9,96 & 1,11 & 1,23 & 0,34 & 1,95 & 93,41 & 374,25 & 2,46 & 4,69 & 0,43 \\
\hline Desvio padrão & 1,28 & 0,18 & 0,22 & 0,14 & 0,26 & 1,16 & 5,16 & 1,40 & 0,48 & 0,14 \\
\hline Variância & 1,63 & 0,03 & 0,05 & 0,02 & 0,07 & 1,36 & 26,60 & 1,95 & 0,23 & 0,02 \\
\hline Mínimo & 6,68 & 0,72 & 0,74 & 0,15 & 1,30 & 90,36 & 358,32 & 1,01 & 3,91 & 0,15 \\
\hline Máximo & 12,74 & 1,63 & 1,94 & 1,37 & 2,75 & 96,14 & 388,36 & 5,86 & 6,20 & 0,65 \\
\hline Assimetria & $-0,04$ & 0,16 & 0,29 & 2,89 & 0,51 & 0,45 & 0,23 & 1,15 & 0,78 & $-0,19$ \\
\hline Curtose & $-0,74 *$ & $-0,16^{*}$ & $0,25^{*}$ & 18,92 & $0,53 *$ & $-0,10$ & 0,32 & $-0,03$ & $-0,07$ & $-1,34$ \\
\hline Grupos & \multicolumn{10}{|c|}{ Método hierárquico } \\
\hline 1 & $11,40 \mathrm{a}$ & $1,23 \mathrm{ab}$ & $1,12 \mathrm{a}$ & $0,42 \mathrm{a}$ & $1,88 \mathrm{~b}$ & $95,34 \mathrm{a}$ & $382,12 \mathrm{a}$ & $4,78 \mathrm{a}$ & $5,48 a b c$ & $0,60 \mathrm{a}$ \\
\hline 2 & $10,01 b c$ & $1,15 b$ & $1,14 \mathrm{a}$ & $0,35 \mathrm{ab}$ & $1,99 \mathrm{ab}$ & $92,85 b$ & $371,40 \mathrm{~b}$ & $1,86 \mathrm{~b}$ & $4,58 b c$ & $0,44 b c$ \\
\hline 3 & $8,71 \mathrm{c}$ & $0,93 \mathrm{c}$ & $1,37 \mathrm{a}$ & $0,28 b$ & $1,85 \mathrm{~b}$ & $93,49 b$ & $375,35 b$ & $1,76 b$ & $4,35 \mathrm{c}$ & $0,27 \mathrm{c}$ \\
\hline 4 & $10,85 b$ & $1,31 \mathrm{a}$ & $1,37 \mathrm{a}$ & $0,35 \mathrm{ab}$ & $2,19 \mathrm{a}$ & $91,97 \mathrm{c}$ & $367,89 \mathrm{c}$ & $2,54 b$ & $4,75 b$ & $0,52 \mathrm{~b}$ \\
\hline
\end{tabular}

G: grupo; U: Umidade \%; CZ: Cinzas \%; P: Proteína total \%; EE: Extrato Etéreo \%; F: Fibra total \%; C: Carboidratos totais \%; VE: Valor Energético (kcal.100g-1); Acidez: Acidez titulável meq NaOH.100-1 $\mathrm{g} ; \mathrm{pH}$ : pH; Aw: Atividade de Água. * distribuição normal por KolmogovSmirnov a $5 \%$.

Rev. Ceres, Viçosa, v. 63, n.2, p. 113-121, mar/abr, 2016 
é, o perfil das médias ponderadas das variáveis, de acordo com cada grupo de farinha (Figura 3). Para o grupo 1, assim como o grupo A, os valores de umidade, carboidratos, valor energético, acidez, $\mathrm{pH}$ e atividade de água situaram-se acentuadamente acima da média relativa dos dados, valor 0.0 (Figura 3), o que o diferenciou dos demais. Ferreira Neto $e t$ al. (2005), também observaram aumento da atividade de água, diretamente proporcional ao tempo de armazenamento das farinhas produzidas na Paraíba, o que interfere no tempo de prateleira do produto, uma vez que, segundo Álvares et al. (2013), existe uma tendência de aumento dos teores de umidade com o período de armazenamento. Além disso, maior acidez pode descaracterizar o produto, já que as farinhas do Grupo Seca geralmente são menos ácidas (Chisté \& Cohen, 2008). Para o grupo 4, os valores de cinzas e de fibra bruta total situaram-se acima da média relativa, associando-o ao grupo C. Carboidratos totais e valor energético ficaram muito abaixo da média relativa, diferentemente dos mesmos parâmetros de farinhas do Estado de São Paulo. Souza \& Menezes (2004) observaram altos teores de carboidratos e fibras, evidenciando o uso de diferentes variedades de mandioca para a produção de farinhas e, possivelmente, alterações no processo de fermentação da massa de mandioca (Chisté \& Cohen, 2011). Já os grupos 2 e 3 mantiveram-se mais estáveis e com variações menos erráticas, como os grupos B e D, com exceção da atividade de água, abaixo da média relativa para o grupo 3 , sendo uma característica benéfica para a farinha, por influenciar seu potencial para o armazenamento prolongado (Ferreira Neto et al., 2004). Chisté \& Cohen (2006) citam que, em características como proteína total e extrato etéreo, pode haver variações entre as amostras de farinha decorrentes das características intrínsecas das raízes da mandioca. Contudo, o teor de umidade, acidez e atividade de água, por exemplo, estão relacionados com o seu processo de fabricação (Chisté \& Cohen, 2010), o que também foi observado por Chisté et al. (2006) e Souza et al. (2008b), na fabricação de farinhas do Grupo Seca.

A distribuição das amostras contidas em cada grupo, de acordo com seu município de origem, pode ser observada na Figura 4. Os grupos 2 e 3, com menores variações, contêm a maioria das amostras obtidas em Mâncio Lima, Cruzeiro do Sul e Rodrigues Alves. Souza et al. (2008a) observaram o mesmo comportamento, indicando que, para este caso, a variabilidade das farinhas não está representada pelo local de produção, uma vez que estes grupos são provenientes de três municípios vizinhos. O grupo 4, com teor elevado de cinzas, é composto principalmente

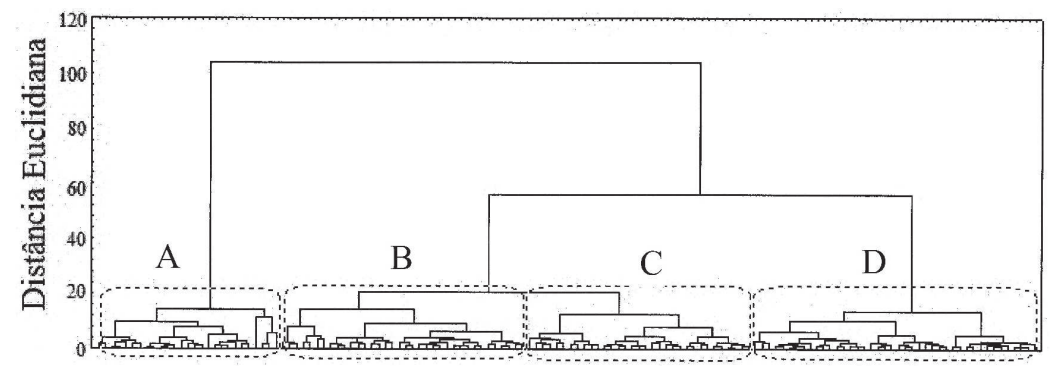

Figura 2: Dendrograma da distância euclidiana, como medida de semelhança entre as características físico-químicas das amostras de farinha, e o método de Ward na construção da hierarquia, com a formação de 4 grupos principais.

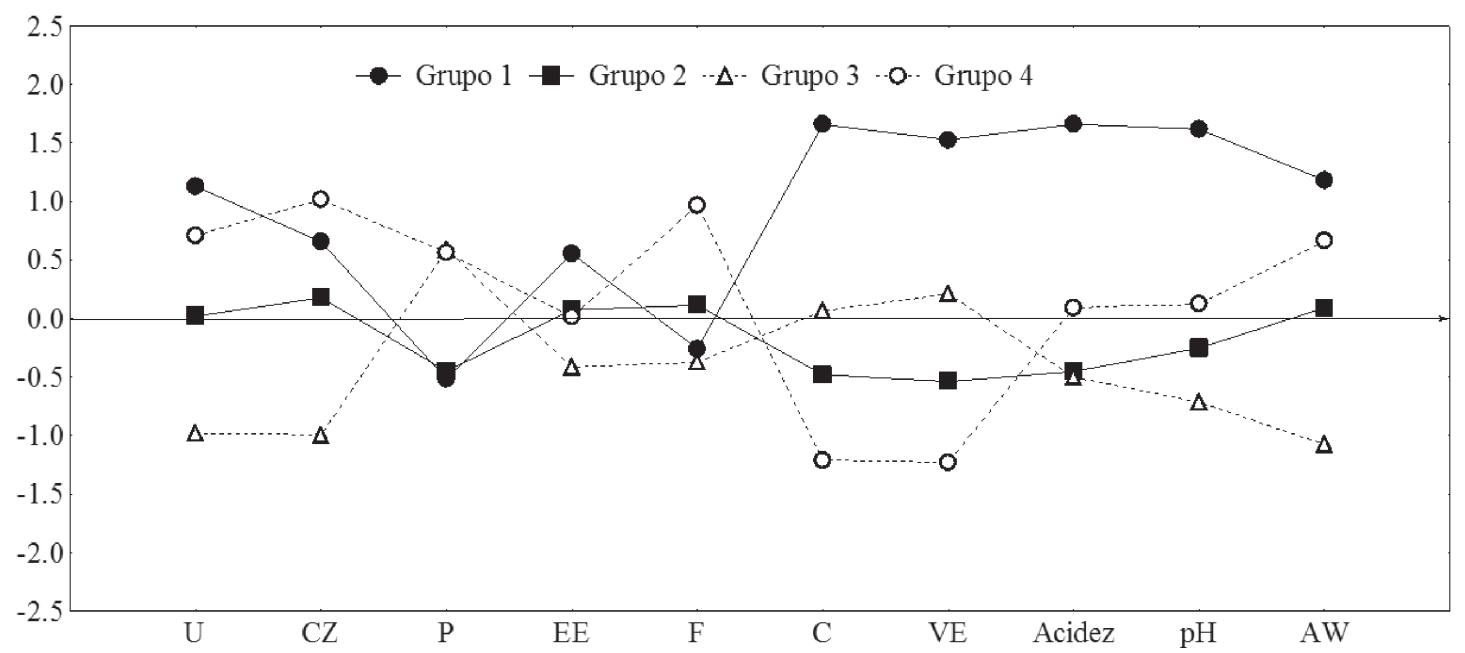

Figura 3: Perfil dos centroides de cada grupo pelo método k-means. U: Umidade \%; CZ: Cinzas \%; P: Proteína total \%; EE: Extrato Etéreo \%; F: Fibra bruta total \%; C: Carboidratos totais \%; VE: Valor Energético (kcal.100g $\left.{ }^{-1}\right)$; Acidez: Acidez titulável meq $\mathrm{NaOH} \cdot 100^{-1} \mathrm{~g}$; pH: pH; Aw: Atividade de Água. 
por amostras de Porto Walter. No entanto, para o grupo 1, verifica-se que o local é predominante. Isto se deve à distância geográfica do município de Marechal Thaumaturgo, em relação aos demais. Neste caso, possivelmente o processo de fabricação utilizado seja distinto, resultando em uma farinha com características diferentes (Velthem \& Katz, 2012), o que pode ter influenciado nos maiores teores de umidade e na atividade de água do produto, em relação aos dos demais municípios (Tabela 1).

O grupo 1, diferentemente da média geral, apresentou acidez significativamente superior à dos demais grupos, classificada como acidez alta $\left(\geq 3,0\right.$ meq NaOH.100 g g $\left.^{-1}\right)$ de acordo com a legislação (Brasil, 2011), o que pode ser resultado de um maior tempo de prensagem da massa de mandioca ralada e de maior exposição desta ao ambiente, o que também foi observado por Chisté \& Cohen (2007; 2011). Quanto ao teor de umidade, embora todos os grupos estejam de acordo com os padrões da legislação vigente, observa-se que houve diferença significativa entre os grupos, com maior teor de umidade para o grupo 1 . Teores elevados de umidade também podem indicar falhas no processamento da farinha, caracterizado pelos processos artesanais, como o tempo de secagem insuficiente (Dias \& Leonel, 2006; Dósea et al., 2010). Além disso, o grupo 1, superior estatisticamente aos demais, apresenta média de atividade de água de 0,60, considerada como limite mínimo para a proliferação de microorganismos (Ferreira Neto et al., 2005; Chisté et al., 2006). Álvares et al. (2013) afirmam que o processo de secagem é fundamental para diminuir a carga microbiana e tornar o produto adequado ao consumo humano. O maior teor médio de cinzas, no grupo 1, embora de acordo com a legislação, pode indicar maior teor de minerais, como também contaminação por material estranho, por falhas em alguma etapa do processamento (Chisté \& Cohen, 2010). Os maiores teores de proteínas foram encontrados nos grupos 3 e 4, porém sem diferença estatísticas dos demais, sendo valores dependentes também da variedade de mandioca utilizada no preparo (Souza et al., 2008b). Quanto ao teor de fibras, todos os grupos estavam de acordo com a legislação (máximo de 2,3\%), com diferenças estatísticas significativas apenas entre os grupos 4 e 3 .
Os valores individuais e acumulados dos autovalores obtidos na análise de componentes principais (ACP) podem ser observados na Tabela 2, onde os três primeiros componentes retêm $73,4 \%$ da variância original dos dados. No primeiro componente principal $(39,9 \%)$, as variáveis que mais influenciaram na discriminação das farinhas foram acidez e $\mathrm{pH}$, seguidas pela atividade de água e umidade. Todas estas variáveis são influenciadas pelo modo de processamento, indicando que a padronização do processo de produção artesanal da farinha de mandioca é muito importante para a qualidade final do produto (Álvares et al., 2013). Para o segundo componente principal $(22,2 \%)$, as variáveis que mais influenciaram foram valor energético e carboidratos totais em contraste com o teor de fibra bruta total. Fiorda et al. (2013) também observaram este comportamento inverso de fibras e carboidratos na extração da fécula de mandioca, em função de variações no processo de extração. E para o terceiro componente $(11,2 \%)$, a variável com maior influência foi teor de proteína total. Já para Souza et al. (2008a), as variáveis mais importantes na discriminação das farinhas de mandioca foram os carboidratos totais e a umidade, retendo mais de $78 \%$ da variância dos dados, o que pode ser explicado pela abrangência amostral menor, englobando apenas as farinhas da região de Cruzeiro do Sul. Segundo Álvares et al. (2013), quando a totalidade de fornecedores é maior, como no caso do centro de comercialização em Rio Branco, há a possibilidade de mistura de farinhas com processos de produção diferentes, o que pode tornar a farinha "fora do tipo", com características variáveis ou fora dos padrões estabelecidos pela legislação.

A projeção dos componentes no plano (Figura 5) mostra a distribuição das amostras, em relação à influência das variáveis. As amostras estão bem discriminadas, principalmente pela influência da acidez, do $\mathrm{pH}$, da atividade de água e da umidade, isto é, amostras mais ácidas são também as mais úmidas e apresentam-se diferentemente das demais. Isso ocorre por influência da umidade no processo de fermentação da massa, aumentando a acidez da farinha (Chisté \& Cohen, 2011). Já a influência do valor energético e dos carboidratos, em contraste com a do teor

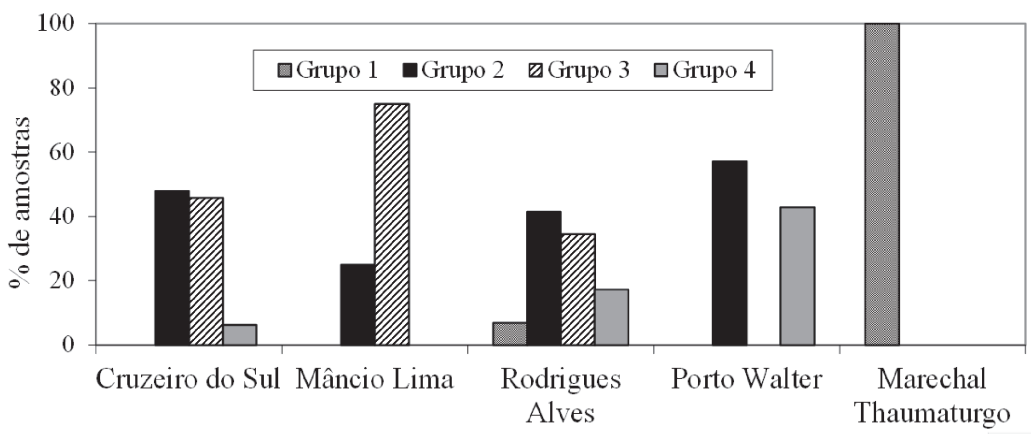

Figura 4: Percentagens de amostras por município, segundo os grupos de farinhas pelo agrupamento k-means.

Rev. Ceres, Viçosa, v. 63, n.2, p. 113-121, mar/abr, 2016 
de proteínas, revela seu comportamento inverso, também observado por Fiorda et al. (2013), distribuído na parte superior e inferior do gráfico, respectivamente.

$\mathrm{O}$ agrupamento das farinhas por município, em percentagem e por amostra, pode ser observado na Tabela 3. As variáveis físico-químicas consideradas relevantes pelo método novamente relacionaram $\mathrm{pH}$ e acidez, com a primeira função de discriminação, contendo $97,1 \%$ da discriminação entre os grupos e a segunda $2,9 \%$.

Estes resultados corroboram os observados pela análise de agrupamento, na qual os municípios Mâncio Lima, Rodrigues Alves e Cruzeiro do Sul não apresentam discriminação definida (Figura 6), provavelmente por causa da proximidade entre eles e da semelhança dos modos de fabricação dos produtores dessa região (Velthem \& Katz, 2012). O maior reflexo desse comportamento pode ser ob- servado nas amostras obtidas em Mâncio Lima, que não apresentam características particulares, sendo consideradas semelhantes às dos municípios vizinhos, em relação às características físico-químicas. Já os municípios de Porto Walter e de Marechal Thaumaturgo apresentaram mais de 85 e $100 \%$ de amostras agrupadas para cada um, respectivamente, indicando novamente a diferenciação físico-química de suas farinhas na região. Para o município de Cruzeiro de Sul, algumas amostras foram designadas como semelhantes, físico-quimicamente, às de Porto Walter, indicando uma possível discrepância pontual na produção dessas casas de farinha. No entanto, algumas amostras de Porto Walter foram designadas como semelhantes às de Cruzeiro do Sul, sugerindo que essas casas de farinhas específicas mantêm os padrões de qualidade dos demais municípios. Já a clara distinção das amostras de Marechal

Tabela 2: Autovalores, variância dos dados originais e autovetores das variáveis e de cada componente principal

\begin{tabular}{lccc}
\hline & CP1 & CP2 & CP3 \\
\hline Autovalores & 3,987 & 2,225 & 1,125 \\
Variância total (\%) & 39,875 & 22,254 & 11,253 \\
Autovalores acumulados & 3,987 & 6,213 & 7,338 \\
Variância acumulada (\%) & 39,875 & 62,128 & 73,381 \\
\hline Variáveis & & Autovetores & $-0,3705$ \\
U & $-0,7061^{*}$ & 0,4814 & 0,2117 \\
CZ & $-0,5816$ & 0,5520 & $-0,7296^{*}$ \\
P & 0,3844 & $-0,0847$ & 0,1886 \\
EE & $-0,4667$ & $-0,0118$ & 0,4312 \\
F & 0,0679 & $0,6418^{*}$ & 0,2275 \\
C & $-0,6472$ & $-0,6640^{*}$ & 0,0267 \\
VE & $-0,5983$ & $-0,7854^{*}$ & $-0,2006$ \\
Acidez & $-0,8455^{*}$ & $-0,1413$ & 0,0488 \\
pH & $-0,8377^{*}$ & 0,0492 & $-0,3069$ \\
AW & $-0,7662^{*}$ & 0,4352 & C
\end{tabular}

CP: componente principal; U: Umidade \%; CZ: Cinzas \%; P: Proteína total \%; EE: Extrato Etéreo \%; F: Fibra total \%; C: Carboidratos totais \%; VE: Valor Energético (kcal.100g-1); Acidez: Acidez titulável meq NaOH.100 ${ }^{-1} \mathrm{~g}$; pH: pH; Aw: Atividade de Água. * maiores autovetores.
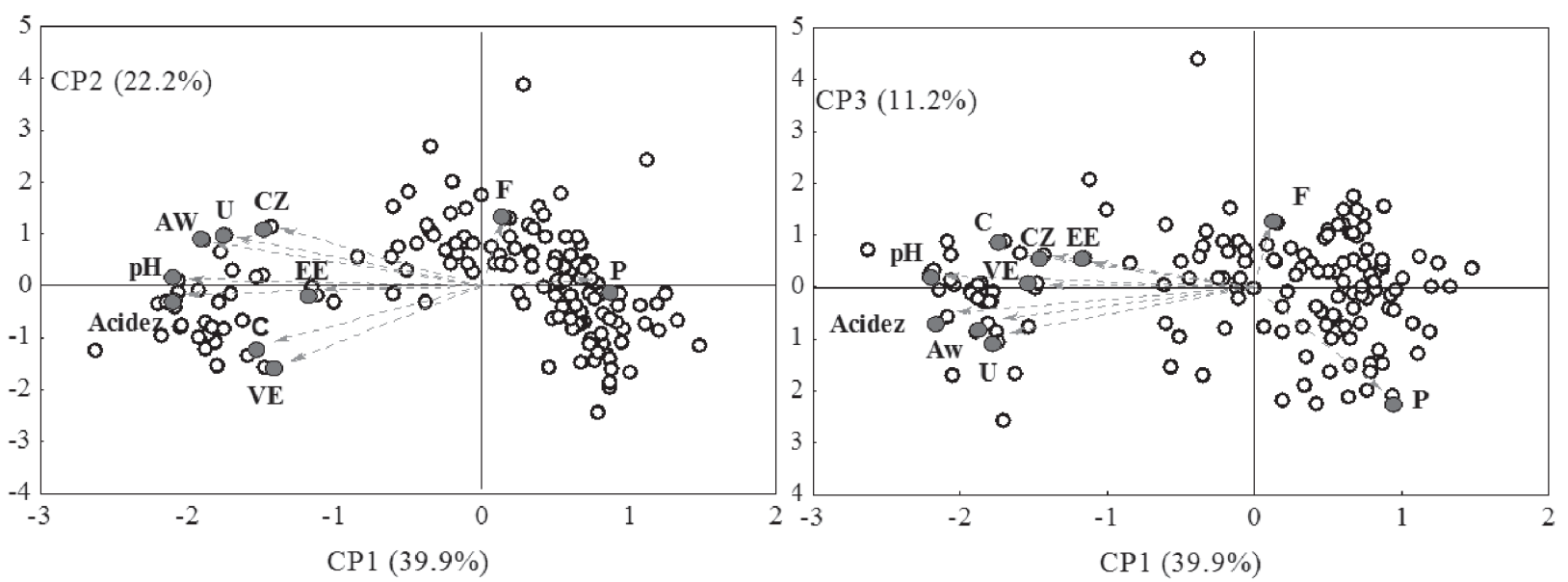

Figura 5: Projeção das variáveis no plano: A. componente principal 1 (CP1) e componente principal 2 (CP2) (variância acumulada de $61,1 \%)$; B. CP1 e CP3 (51.1\%). 
Tabela 3: Agrupamento correto das amostras de farinha de mandioca por município, segundo a análise discriminante

\begin{tabular}{lrccccc}
\hline & \% & CruzSul & ManLin & RodAlv & PorWal & MalTha \\
\hline CruzSul & 56,2 & 27 & 0 & 16 & 5 & 0 \\
ManLin & 0,0 & 13 & 0 & 3 & 0 & 0 \\
RodAlv & 34,5 & 18 & 0 & 10 & 1 & 0 \\
PorWal & 85,7 & 3 & 0 & 0 & 18 & 0 \\
MalTha & 100,0 & 0 & 0 & 0 & 0 & 24 \\
Total & 57,2 & 61 & 0 & 29 & 24 & 24 \\
\hline
\end{tabular}

CruzSul: Cruzeiro do Sul; ManLin: Mâncio Lima; RodAlv: Rodrigues Alves; PorWal: Porto Walter; MalTha: Marechal Thaumaturgo.

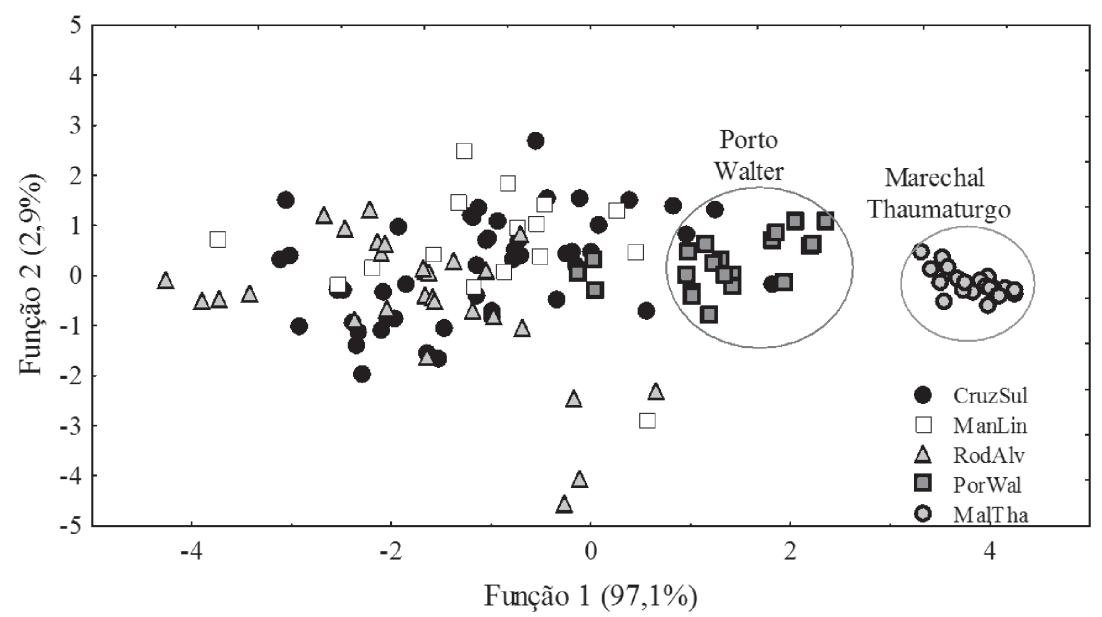

Figura 6: Gráfico contrastando as funções discriminantes das amostras de farinhas, geradas a partir das variáveis físico-químicas. CruzSul: Cruzeiro do Sul; ManLin: Mâncio Lima; RodAlv: Rodrigues Alves; PorWal: Porto Walter; MalTha: Marechal Thaumaturgo.

Thaumaturgo, baseada nos resultados encontrados, sugere uma revisão em seu sistema de produção, em relação às farinhas dos demais municípios.

\section{CONCLUSÕES}

As farinhas produzidas no Território da Cidadania do Vale do Juruá, Acre estão de acordo com os padrões de qualidade previstos pela legislação brasileira.

Os municípios de Porto Walter e, principalmente, de Marechal Thaumaturgo produzem farinhas de qualidade discrepante das demais produzidas na região estudada.

As variáveis de maior influência na classificação da farinha de mandioca para esta região são acidez, $\mathrm{pH}$, atividade de água e umidade, indicando que o modo de fabricação, associado à distribuição geográfica dos municípios, tem grande influência na qualidade da farinha produzida.

\section{AGRADECIMENTOS}

Os autores agradecem aos técnicos da Secretaria de Extensão Agroflorestal e Produção Familiar (SEAPROF) e ao Ministério da Agricultura, Pecuária e Abastecimento (MAPA), pelo apoio no georeferenciamento e, aos produtores familiares do TCVJ, pelo acolhimento dos técnicos em suas residências.

\section{REFERÊNCIAS}

Álvares VS, Costa DA, Felisberto FAV, Silva SF \& Madruga ALS (2013) Atributos físicos e químicos da farinha de mandioca artesanal em Rio Branco, Acre. Caatinga, 26:50-58.

AOAC - Association of Official Analytical Chemists (1995) Official methods of analysis of the AOAC International. $16^{\mathrm{a}}$ ed. Arlington, AOAC International. 1025p.

Brasil (2001) Resolução RDC n. 12, de 10 de janeiro de 2001. Aprova o Regulamento Técnico sobre padrões microbiológicos para alimentos. Diário oficial da República Federativa do Brasil, 10/01/2001. Seção 1, p. 45-53.

Brasil (2011) Instrução Normativa $n^{\circ} 52$, de 07 de novembro de 2011. Regulamento técnico da farinha de mandioca. Diário Oficial da República Federativa do Brasil, 08/11/2011, Seção 1, p.18-20.

Cereda MP \& Vilpoux O (2010) Metodologia para divulgação de tecnologia para agroindústrias rurais: exemplo do processamento de farinha de mandioca no Maranhão. Revista Brasileira de Gestão e Desenvolvimento Regional, 6:219-250.

Chisté RC \& Cohen KO (2006) Estudo do Processo de Fabricação da Farinha de Mandioca. Belém, Embrapa Amazônia Oriental. 76p. (Documentos, 267).

Chisté RC \& Cohen KO (2007) Comportamento da acidez total e amido na produção da farinha de mandioca do grupo seca. Revista Brasileira de Tecnologia Agroindustrial, 1:17-25.

Chisté RC \& Cohen KO (2008) Determinação de cianeto total nas farinhas de mandioca do grupo seca e d'água comercializadas na cidade de Belém-PA. Revista Brasileira de Tecnologia Agroindustrial, 2:96-102. 
Chisté RC \& Cohen KO (2010) Caracterização fisico-química da farinha de mandioca do grupo d'água comercializada na cidade de Belém, Pará. Revista Brasileira de Tecnologia Agroindustrial, 4:91-99.

Chisté RC \& Cohen KO (2011) Influência da fermentação na qualidade da farinha de mandioca do grupo d'água. Acta Amazonica, 41:279-284.

Chisté RC, Cohen KO, Mathias EA \& Ramos Junior AGA (2006) Qualidade da farinha de mandioca do grupo seca. Ciência e Tecnologia de Alimentos, 26:861-864.

Dias LT \& Leonel M (2006) Caracterização físico-química de farinhas de mandioca de diferentes localidades do Brasil. Ciência e Agrotecnologia, 30:692-700.

Dósea RR, Marcellini PS, Santos AA, Ramos ALD \& Lima AS (2010) Qualidade microbiológica na obtenção de farinha e fécula de mandioca em unidades tradicionais e modelo. Ciência $\mathrm{Ru}$ ral, 40:441-446.

Ferreira Neto CJ, Nascimento EM, Figueirêdo RMF \& Queiroz AJM (2004) Microbiologia de farinhas de mandioca (Manihoit esculenta Crantz) durante o armazenamento. Ciência Rural, 34:551-555.

Ferreira Neto CJ, Figueirêdo RMF \& Queiroz AJM (2005) Avaliação sensorial e da atividade de água em farinhas de mandioca temperadas. Ciência e Agrotecnologia, 29:795-802.

Fiorda FA, Soares Junior MS, Silva FA, Souto LRF \& Grossmann MVE (2013) Farinha de bagaço de mandioca: aproveitamento de subproduto e comparação com fécula de mandioca. Pesquisa Agropecuária Tropical, 43:408-416.
Instituto Adolfo Lutz (2008) Normas analíticas do Instituto Adolfo Lutz: métodos físico-químicos para análise de alimentos. $4^{\mathrm{a}}$ ed. São Paulo, Instituto Adolfo Lutz. 1020p.

Mingoti SA (2007) Análise de dados através de métodos de estatística multivariada: uma abordagem aplicada. Belo Horizonte, UFMG. 297p.

Oluwamukomi MO, Oluwalana IB \& Akinbowale OF (2011) Physicochemical and sensory properties of wheat-cassava composite biscuit enriched with soy flour. African Journal of Food Science, 5:50-56.

Souza JML, Negreiros JRS, Álvares VS, Leite FMN, Sousa ML, Reis FS \& Felisberto FAV (2008a) Variabilidade físico-química da farinha de mandioca. Ciência e Tecnologia de Alimentos, 28:907-912.

Souza JML, Álvares VS, Leite FMN, Sousa ML, Reis FS \& Felisberto FAV (2008b) Caracterização físico-química de farinhas de mandioca oriundas do município de Cruzeiro do Sul - Acre. Ciências Agrárias e Engenharias, 14:43-49.

Souza ML \& Menezes HC (2004) Processamento de amêndoa e torta de castanha-do-brasil e farinha de mandioca: parâmetros de qualidade. Ciência e Tecnologia de Alimentos, 24:120-128.

Statsoft INC (1995) Statistica for Windows - computer program manual. Tulsa, StatSoft. 322p.

Velthem LH \& Katz E (2012) A 'farinha especial': fabricação e percepção de um produto da agricultura familiar no vale do rio Juruá, Acre. Boletim do Museu Paraense Emílio Goeldi, 7:435456. 